WHITE PAPER FOR PLANETARY AND ASTROBIOLOGY SCIENCE STRATEGY

\title{
Synergy between Ice Giant and Exoplanet Exploration: The Solar System's Planets “As Exoplanets"
}

\author{
Authors: \\ Jonathan Fortney, University of California, Santa Cruz \\ jfortney@ucsc.edu, 831-531-7568 \\ Mark Marley, NASA Ames Research Center \\ Laura Mayorga, Harvard/Smithsonian Center for Astrophysics \\ Abigail Rymer, Johns Hopkins Applied Physics Laboratory

\section{Co-Signers:} \\ Lynnae C. Quick, NASA Goddard Space Flight Center \\ Kevin Stevenson, Johns Hopkins Applied Physics Laboratory \\ Mark Hofstadter, Jet Propulsion Laboratory
}


Given the uniqueness of the Ice Giants as solar system targets, and the longevity of the cruise phase and orbital tour, an Ice Giant mission has the potential to make major impacts to exoplanetary science. In particular there is significant novel science in viewing our solar system's planets "as exoplanets." This complements detailed characterization of the Ice Giant planets themselves.

Exoplanet targets of future direct imaging missions will be viewed as mere points of light. (See Figure 1.) Scattered light from these distant targets data will be at a range of phase angles, and at wavelengths from the ultraviolet to the near infrared. In thermal emission as well, we would only have access to hemispherically-averaged flux. Viewing our solar system planets from afar, such as during a journey to Neptune or Uranus, will allow us to examine their total hemisphericallyaveraged planetary fluxes as a function of orbital phase and of time. This is an essential step in comparing such observations to our "ground truth" understanding of these planets from decades of solar-system planetary science. This science can be done on the "cruise phase" to, for instance, the proposed Odyssey mission concept to Neptune, or other mission concepts.

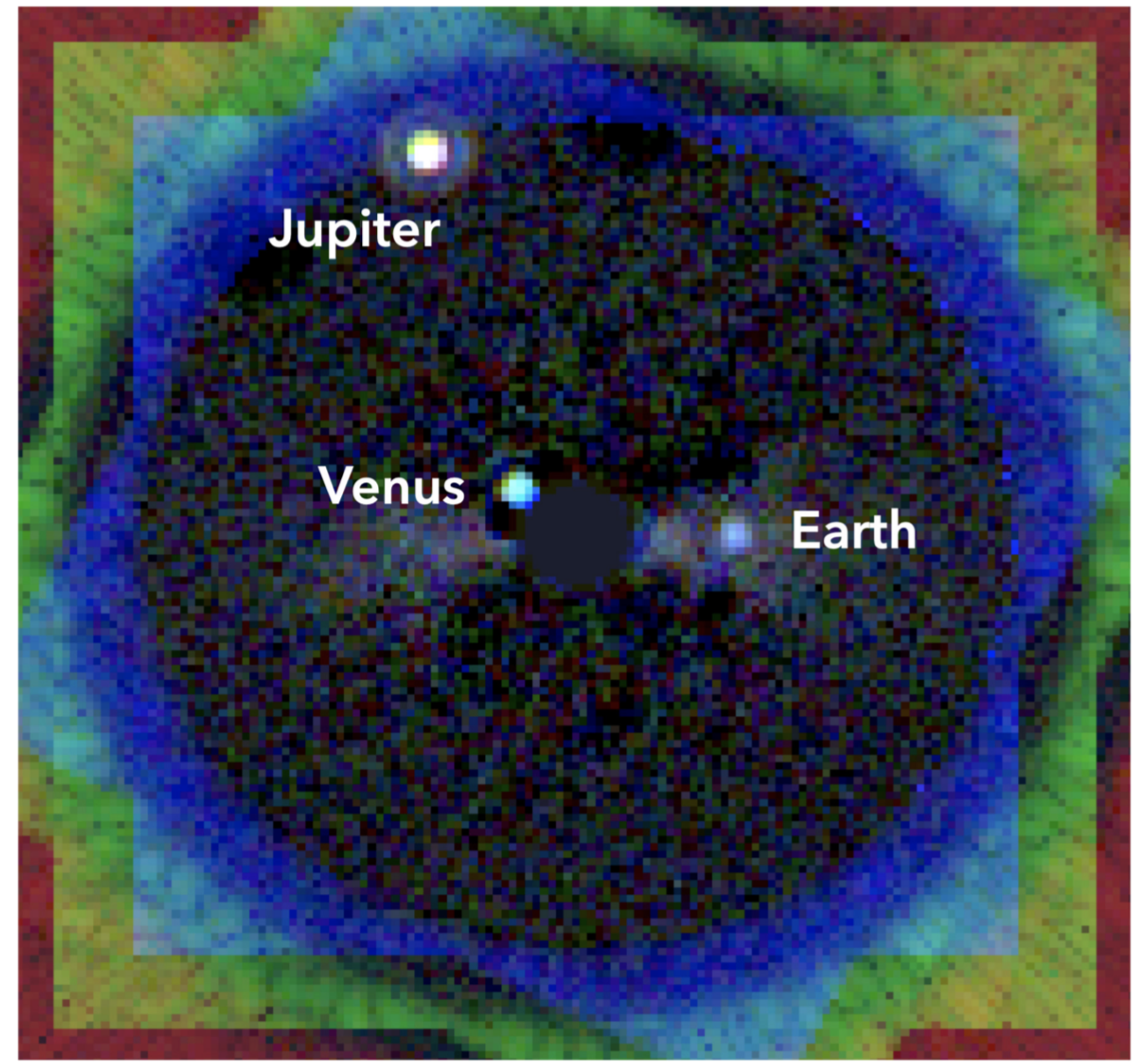

Figure 1: Simulated image of the solar system as would be seen from 10pc away by the LUVOIRA space telescope concept now being considered by the Astro2020 decadal review. The planets here are seen at about 90 degree, pole-on, phase angle, but the typical phase angles will be 60 to 100 degrees. 
This science is important both for gas giant planets and for terrestrial worlds. Already, we have data on time variable thermal emission from young hot gas giants and brown dwarfs (Apai et al. 2017). A major science theme over the past 5-10 years has been in trying to understand the physical mechanisms that drive this variability (Morley et al. 2014, Robinson \& Marley, 2014). Obtaining long-time duration thermal infrared data sets on the character of variability in thermal emission from Jupiter, Saturn, Uranus, and Neptune serves as an essential complement.

Furthermore, a spacecraft offers a unique vantage point that cannot be accessed from Earth: namely the ability to determine the phase-angle dependence of reflectivity and scattering. Deriving ground-truth scattered light phase curves at phases not accessible to Earth is important for testing giant planet atmosphere models that will be used to interpret exoplanet data. Kepler was able to study the low-phase disk-integrated variability on Neptune in visible light to understand the longevity of clouds and their relation to the zonal winds (Simon et al. 2016), but a long cruise phase mission will be able to provide this from multiple vantage points for all the giant planets. Discerning variability at high phase angles is important because the relative importance of particle and gas opacity varies both with altitude and with observer phase angle. Thus partial phase variability can be quite different from full phase. Cassini observed one rotation of Jupiter from Saturn orbit at 60 degrees phase, but this limited baseline precludes understanding of longer timescale variability that might be seen in reflected light.

Missions to the outer solar system can make a large impact on the prospects for characterizing and mapping potentially Earth-like planets. In the first two years of the mission, look-back observations to Venus and Earth can characterize their scattered light and thermal emission at a variety of wavelengths and phase angles, to understand our ability to determine atmospheric abundances and cloud coverage, and for Earth to monitor daily rotational variability during several seasons. In addition, specular reflection "glint" off of oceans has been suggested as a hallmark for water on rocky exoplanets (Robinson et al. 2010), and this glint signature can also be investigated. Since Earth and Venus are too close to the Sun at later stages of the cruise, this science is best done at the start of the cruise phase. Similar giant planet observations can be conducted at a variety of phase angles later during the journey.

Recent examples of how a full disk imaging set can be used in aid of exoplanet science can be found in Mayorga et al. (2016, 2020/in prep). In Mayorga et al. (2016) Jupiter scattered light phase curves from the Cassini flyby were used to show strong deviations from often assumed Lambertian scattering, as a function of phase angle and wavelength. This data is essential in developing a new generation of exoplanet atmosphere models that will be used to interpret the spectra of directing imaged exoplanets. A longer term effort during a cruise to Uranus or Neptune could also investigate variability in these phase functions.

Mayorga et al. (2020, in prep) focused on a complementary science case -- turning scattered light data into surface brightness maps for terrestrial worlds. In Figure 2 we reproduce a key figure from Mayorga et al. (2020) below, using Cassini flyby data for Jupiter's moon Io. Panels A to F, counterclockwise from upper-right, show how scattered light measurements with orbital phase can be turned into a map of Io surface brightness. This is similar in character to an albedo map that was made for the Earth, based on 24 hours of reflectance data at fixed orbital phase, from NASA's Deep Impact mission looking back to Earth (Cowan et al. 2009). Characterizing extrasolar 
terrestrial planets will be an exceptionally difficult undertaking. Improving our modeling frameworks and methodology with comparison to solar system data sets taken at partial phases is absolutely essential in building confidence that our inferences will be correct.
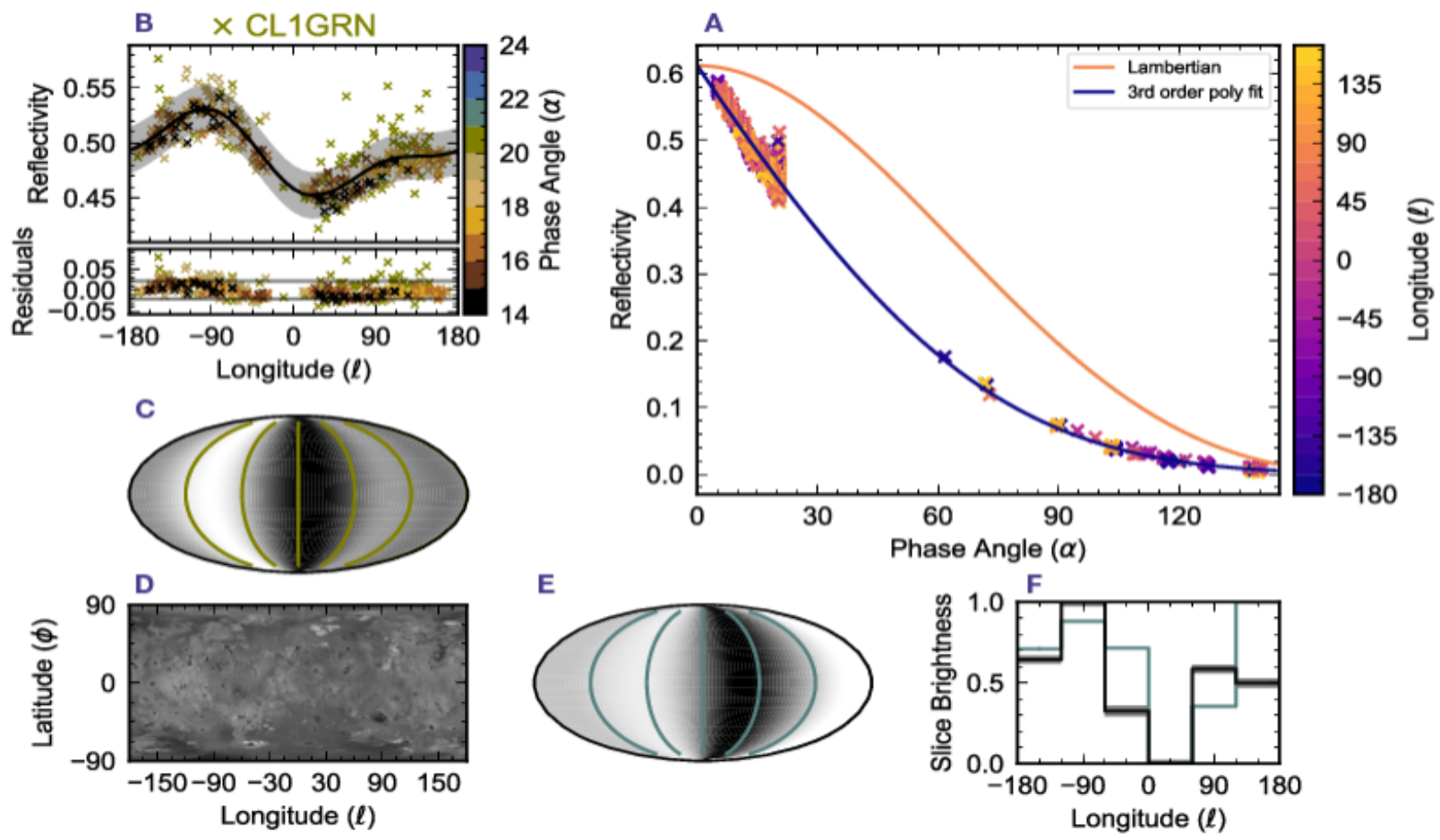

Figure 2: Io "as an exoplanet" as seen through Cassini's GRN filter during the flyby to Saturn. A (upper right): The reflectivity of Io as a function of phase angle as fit by a 3rd order polynomial. Io is not well fit by a Lambertian phase function. B (upper left): The reflectivity of Io as a function of planetocentric latitude at low phase angles as fit by an "orange slice" brightness model of the surface. C: The best fit orange slice brightness model. D: The USGS astrogeology map of Io. The map is likely not a true measure of reflectivity or albedo as it is an image mosaic of Galileo and Voyager data taken at various observational geometries, commonly using a clear filter, but with other filters used at higher resolution. E: The USGS map sliced for comparison with the orange slice brightness model. F: The comparison of individual slice brightnesses between the best fit orange slice brightness model and the USGS map. Adapted from Mayorga et al. in prep.

We advocate for the deliberate inclusion of instrument suites to facilitate these observations - that might be outside the purview of the main mission but address level one science in other disciplines. That said, much of the needed instrumentation would already exist as part of a comprehensive (Cassini-like) Ice Giant flagship (e.g. Rymer et al., PMCS study 2020) moving in wavelength from the UV Imaging Spectrograph, to the Multispectral Narrow Angle Camera, to the Visible-Near IR Camera, and the Thermal IR Imager, would all be important instruments to bring to bear. Having the smallest sun-avoidance angle possible ( $<10$ degrees) would maximize the amount of data that could be gathered, as for the vast majority of the cruise phase the Sun-Earth and Sun-Venus separations would be small. We do not anticipate that data rates will be large, but if needed, detectors should be able to read out or store custom subarray regions of the detector that contain 
planet data. Pointing stability will be important in obtaining precise measurements, though it probably won't have to be at the subpixel level as with Hubble and Spitzer.

Furthermore, comprehensive characterization of the ice giant planets themselves with all of these instruments, and an entry probe, would yield a detailed understanding of the planets. The occurrence rate of exoplanets rises sharply as sizes just smaller than the Ice Giants, with planets between the size of Earth and Neptune the most common yet found. Having "ground-truth" measurements for the Ice Giants is important for building a comprehensive theory for the origin and evolution of these "sub-Neptune" exoplanets. An observation of particular interest to exoplanets, which may not naturally come out of already planned comprehensive characterization of the Ice Giants, would be UV-to-NIR scattered light from the poles, as that is a particularly likely orientation for a future exoplanet direct imaging mission, as shown in Figure 1.

\section{References}

Apai, D., Karalidi, T., Marley, M. S., Yang, H., Flateau, D., Metchev, S., Cowan, N. B., Buenzli, E., Burgasser, A. J., Radigan, J., Artigau, E., \& Lowrance, P. (2017), "Zones, spots, and planetary-scale waves beating in brown dwarf atmospheres," Science, 357, 683.

Cowan, N. B., Agol, E., Meadows, V. S., Robinson, T., Livengood, T. A., Deming, D., Lisse, C. M., A'Hearn, M. F., Wellnitz, D. D., Seager, S., Charbonneau, D., \& EPOXI Team (2009), "Alien Maps of an Ocean-bearing World," The Astrophysical Journal, 700, 915.

Mayorga, L. C., Jackiewicz, J., Rages, K., West, R. A., Knowles, B., Lewis, N., \& Marley, M. S. (2016), "Jupiter's Phase Variations from Cassini: A Testbed for Future Direct-imaging Missions," The Astronomical Journal, 152, 209.

Morley, C. V., Marley, M. S., Fortney, J. J., \& Lupu, R. (2014), "Spectral Variability from the Patchy Atmospheres of T and Y Dwarfs," The Astrophysical Journal, 789, L14.

Robinson, T. D., Meadows, V. S., \& Crisp, D. (2010), "Detecting Oceans on Extrasolar Planets Using the Glint Effect," The Astrophysical Journal, 721, L67.

Robinson, T. D., \& Marley, M. S. (2014), "Temperature Fluctuations as a Source of Brown Dwarf Variability," The Astrophysical Journal, 785, 158.

Simon, A. A., Rowe, J. F., Gaulme, P., Hammel, H. B., Casewell, S. L., Fortney, J. J., Gizis, J. E., Lissauer, J. J., Morales-Juberias, R., Orton, G. S., Wong, M. H., \& Marley, M. S. (2016), "Neptune's Dynamic Atmosphere from Kepler K2 Observations: Implications for Brown Dwarf Light Curve Analyses," The Astrophysical Journal, 817, 162. 